\title{
Confocal bioluminescence imaging for living tissues with a caged
} substrate of luciferin

Mitsuru Hattori $^{1}$, Genki Kawamura ${ }^{1}$, Ryosuke Kojima ${ }^{2}$, Mako kamiya ${ }^{3}$, Yasuteru Urano ${ }^{2,3}$ \& Takeaki Ozawa ${ }^{* 1}$

1. Department of Chemistry, School of Science, The University of Tokyo, 7-3-1 Bunkyo-ku, Hongo, Tokyo 113-0033, Japan

2. Graduate School of Pharmaceutical Sciences, The University of Tokyo, 7-3-1, Bunkyo-ku, Hongo, Tokyo, 113-0033, Japan

3. Graduate School of Medicine, The University of Tokyo, 7-3-1 Bunkyo-ku, Hongo, Tokyo 113-0033, Japan

*Correspondence should be addressed to T. O.

Email: ozawa@chem.s.u-tokyo.ac.jp

Tel.: 81-3-5841-4351

Fax: 81-3-5802-2989 
Table of contents

Figure S1. Structure and localization of NanoLuc _..P3

Figure S2. Scheme of multi-pinhole arrays $\quad \ldots$ P4

Figure S3. Resolution capacity of the imaging system $\quad$..P5

Figure S4. Photoreaction of caged furimazine using multi-pinhole arrays _..P6

Figure S5. Pseudo-color images of bioluminescence from living cell layers

coated with extracellular matrixes $\quad$..P8

Figure S6. Scheme of the construct and fluorescence images of extracellular matrix

...P9

Figure S7. Fluorescence images of EGFP in an epidermal tissue $\quad$..P10

Scheme S1. Synthesis of caged furimazine $\quad$..P11 
A

\section{Pro acrosin}

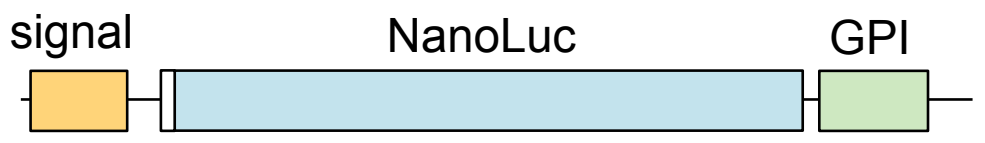

B

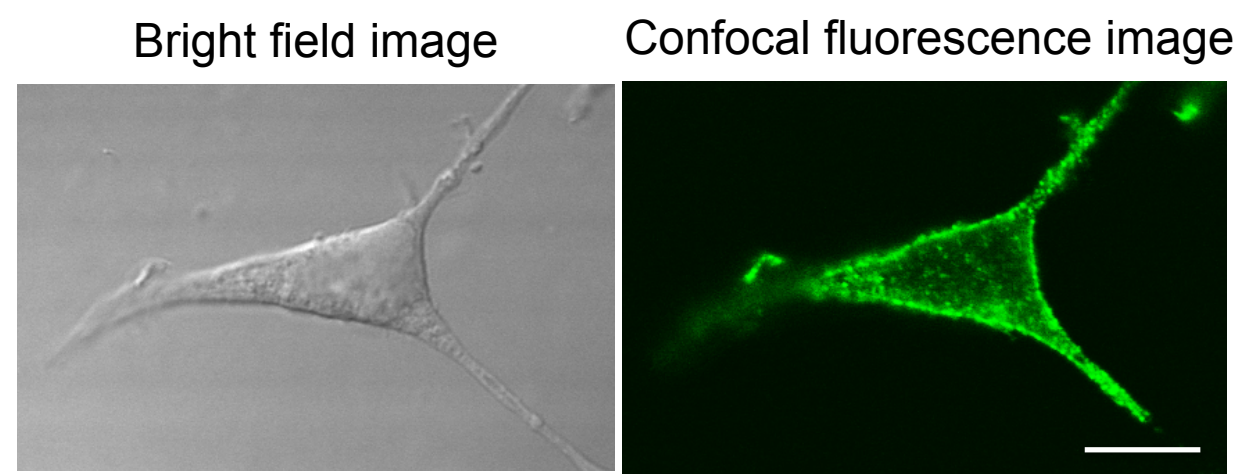

Figure S1. Structure and localization of NanoLuc. (A) Schematic model of NanoLuc with Proacrosin signal and GPI anchor. The proacrosin signal was connected with NanoLuc by "KLT" amino acid sequences. GPI anchor was connected with NanoLuc by "GGGSA". White box shows the epitope myc tag. (B) Localization of NanoLuc in NIH3T3 cell. Bright field and confocal fluorescence images of an immunostained sample are shown. Scale bar, 10 $\mu \mathrm{m}$. 
A

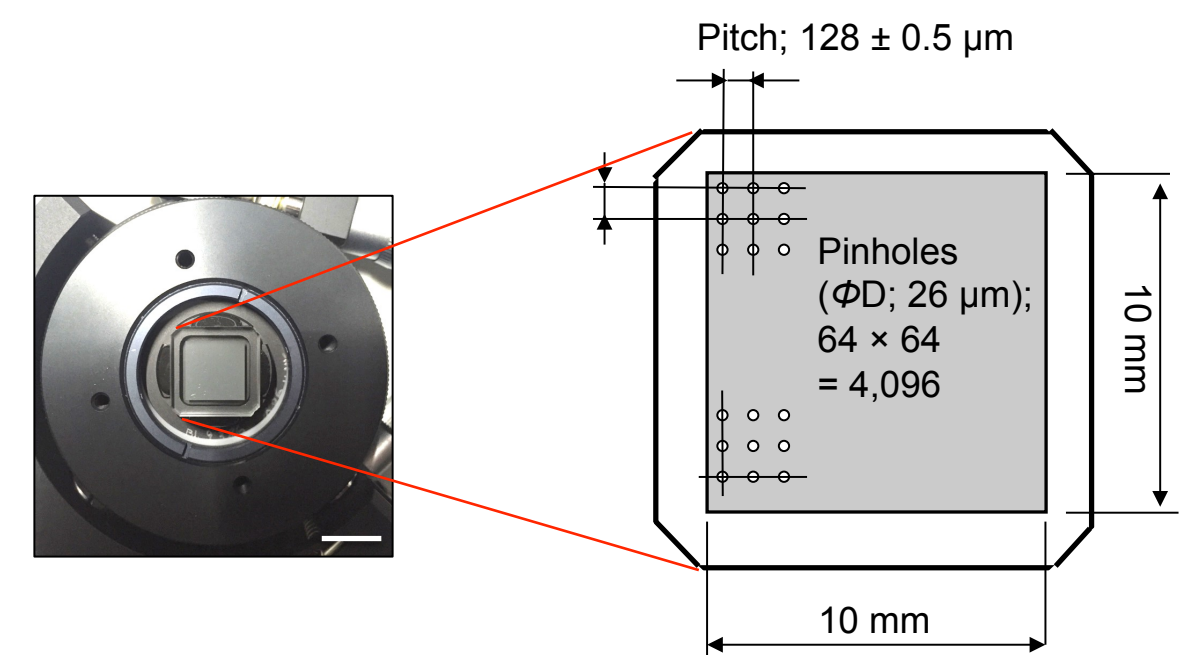

B

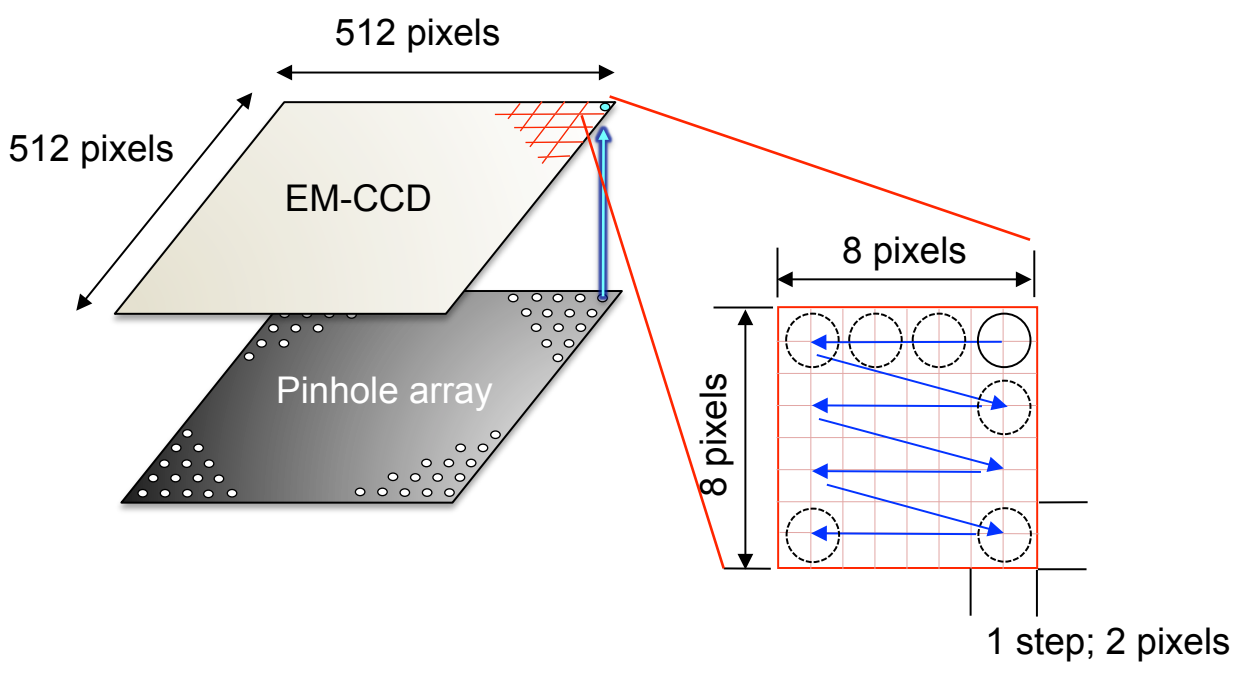

Total; 64 holes $\times 8$ pixels $=512$ pixels

Figure S2. Scheme of multi-pinhole arrays. (A) Detailed structure of the multi-pinhole arrays. Multi-pinhole arrays installed in the new imaging system are presented at left. Scale bar, 10 $\mathrm{mm}$. The array contains 4,096 pinholes (right panel). (B) Schematic model of the scanning steps of the multi-pinhole array. Light that passes through each pinhole is collected on $2 \times 2$ pixels of the CCD camera. A single pinhole covers the $8 \times 8$ pixel area through 16 steps. 
A
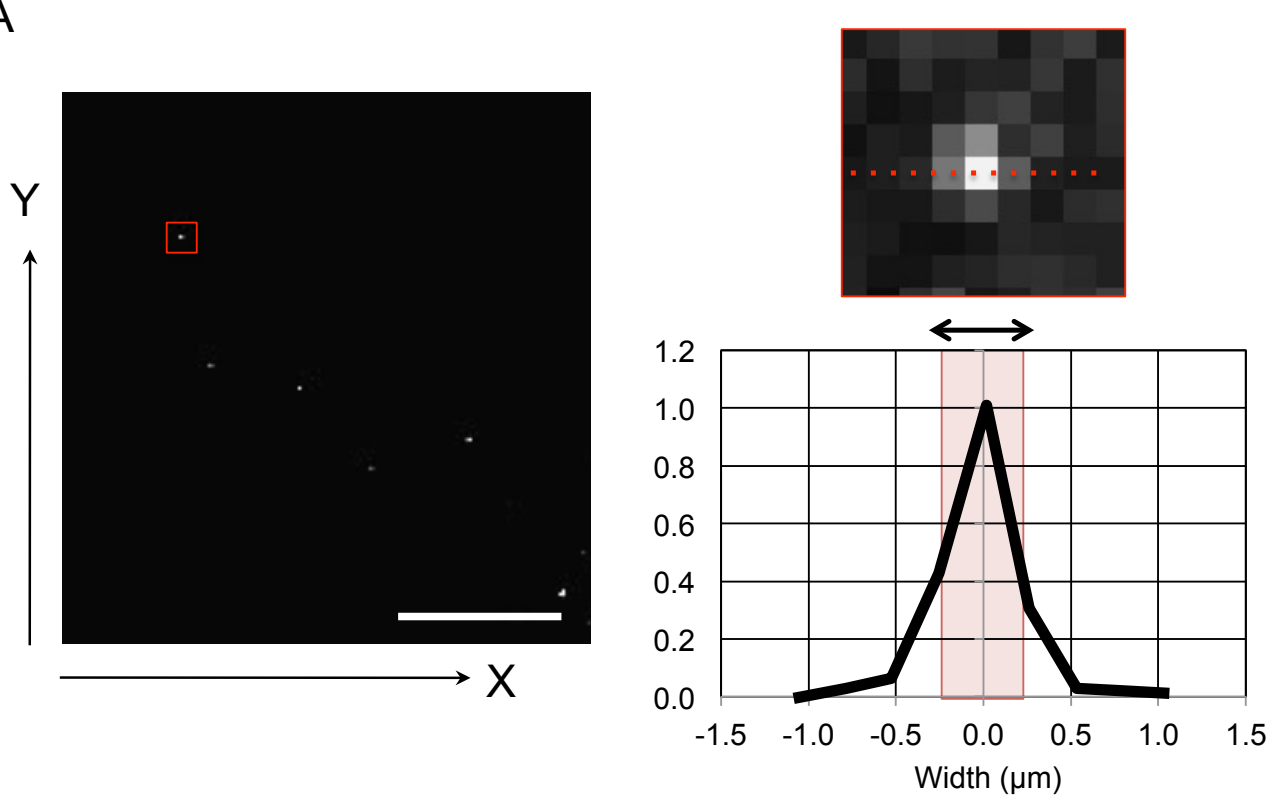

B
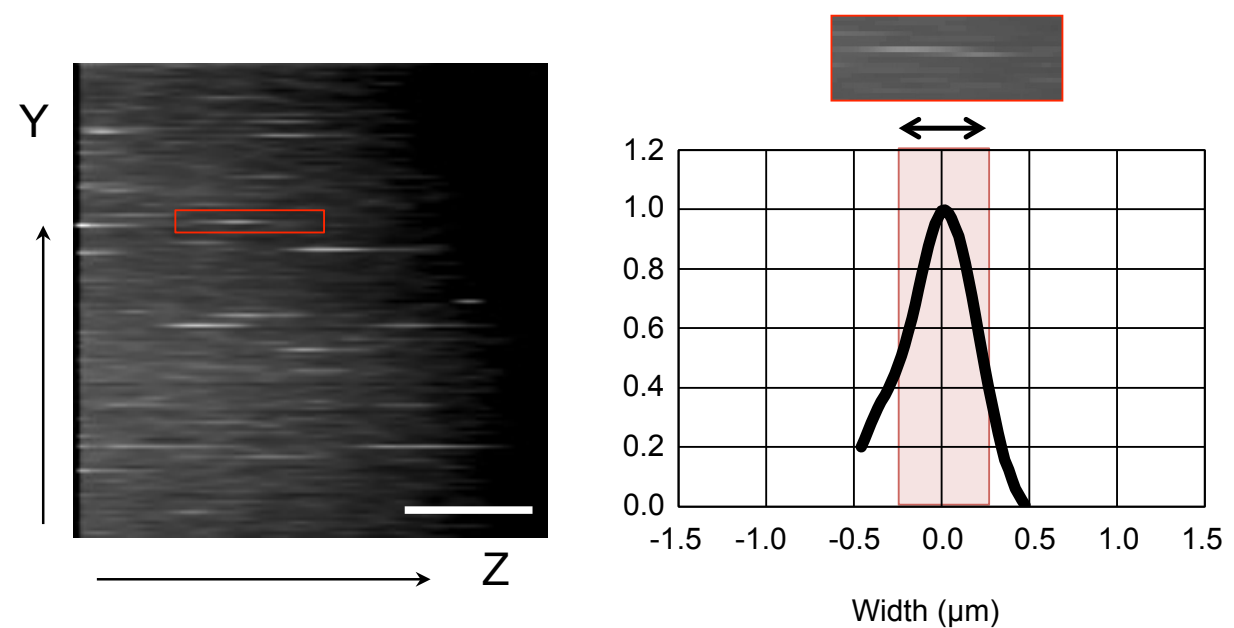

Figure S3. Resolution capacity of the imaging system. Fluorescence beads embedded in resin were observed using the imaging system. We measured the fluorescence spot size in the integrated image. The bead diameter was $0.1 \mu \mathrm{m}$. (A) Calculation of the XY resolution. An enlarged image of a region (open red box) is shown at left. The normalized fluorescence intensity in the graph was measured along the red broken line. The red region in the graph shows the region within half-band width. Scale bar, $1 \mu \mathrm{m}$. (B) Resolution in the depth direction (z). Scale bar, $1 \mu \mathrm{m}$. 
A

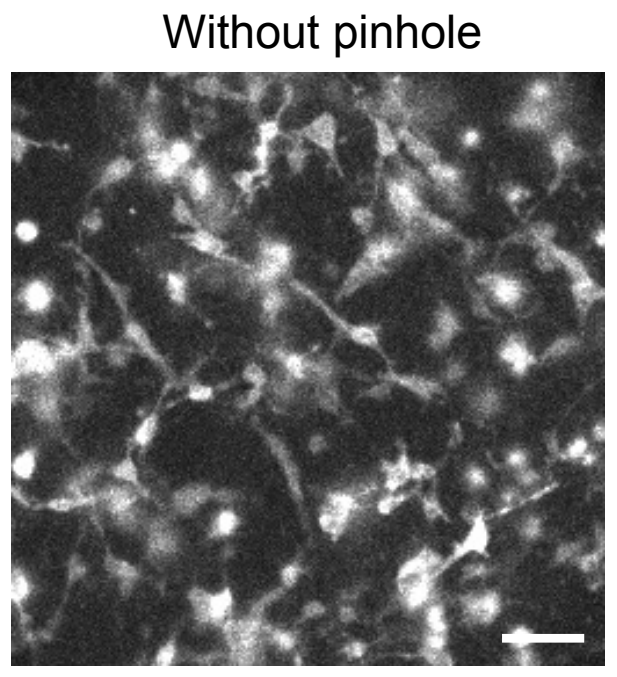

Through pinhole

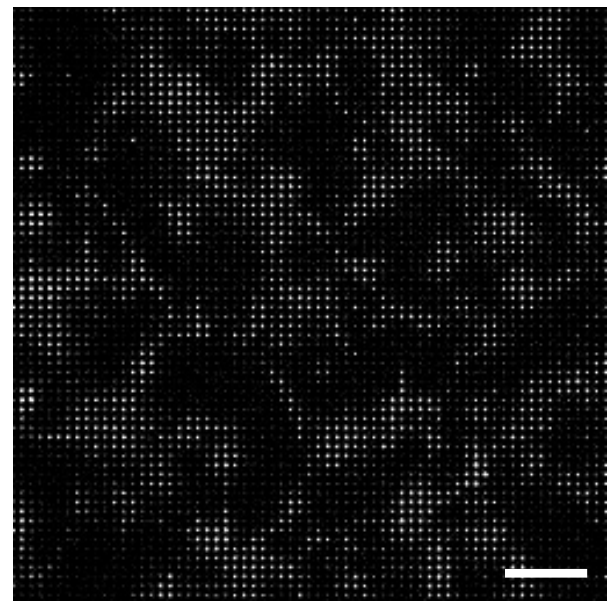

B

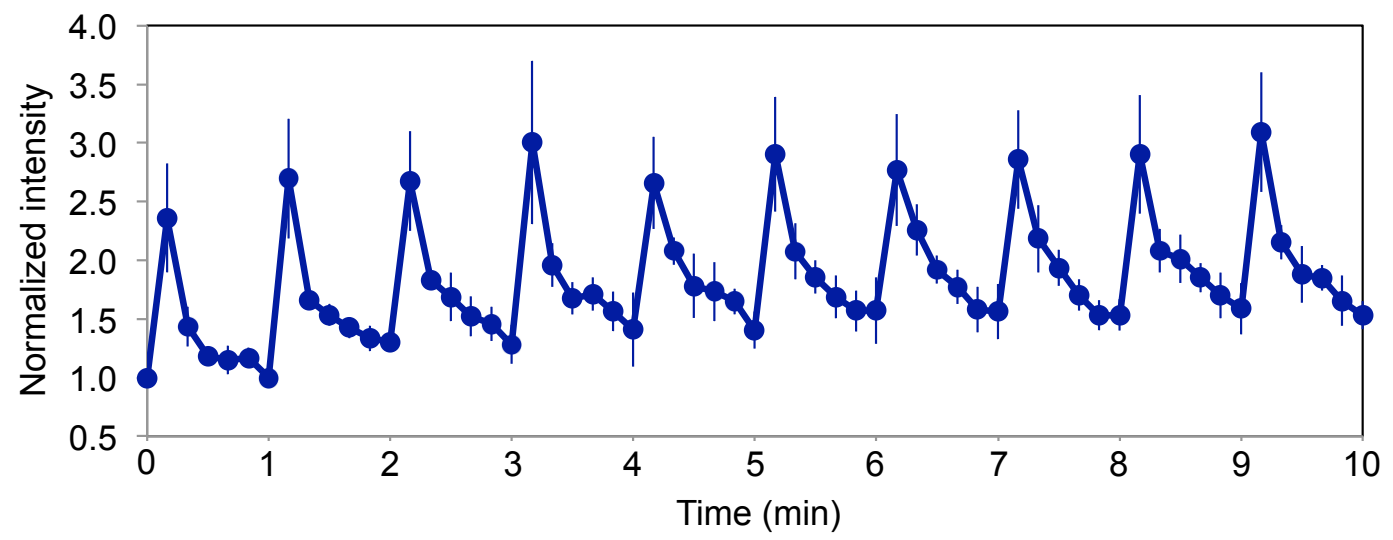

C
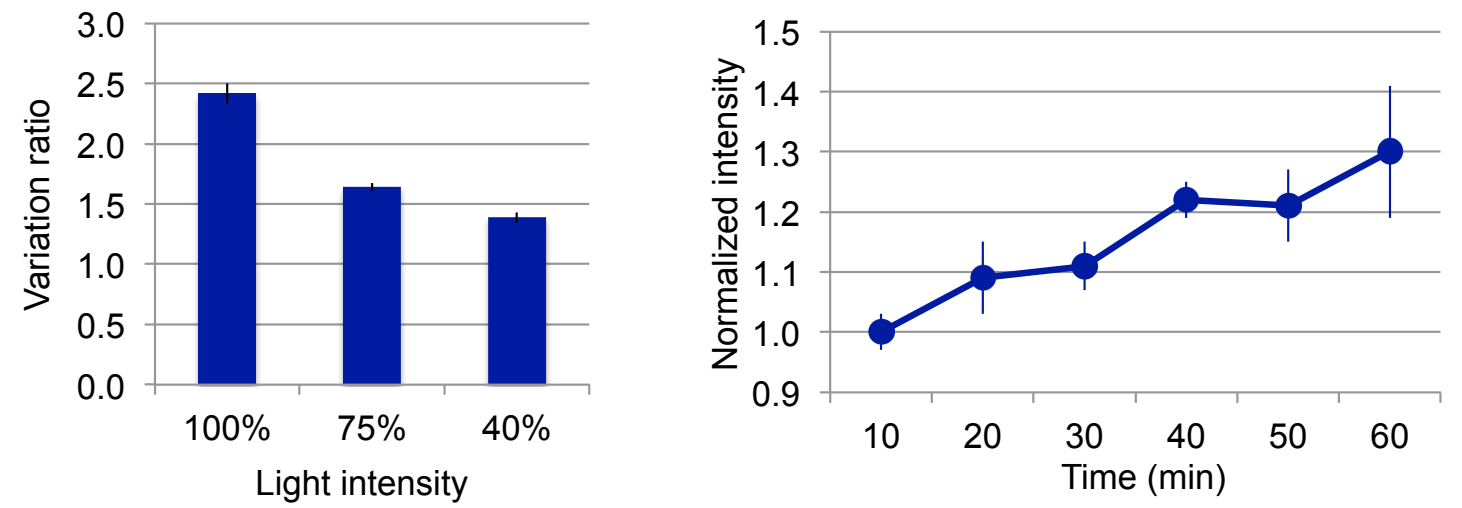

Figure S4. Photoreaction of caged furimazine using multi-pinhole arrays: (A) Bioluminescence images of the NIH3T3 cells obtained using the bioluminescence imaging system. The left image was taken without a pinhole. The right image shows a snapshot 
through the pinhole, which was taken after the irradiation. Each exposure time was $10 \mathrm{~s}$. Scale bar, $50 \mu \mathrm{m}$. (B) Temporal variation of bioluminescence with repetitive light irradiation. Bioluminescence from arbitrary cell regions in the pinhole image of Figure S4A was measured using $10 \mathrm{~s}$ exposure. The irradiation was done at $1 \mathrm{~min}$ intervals. Average normalized bioluminescence intensities are shown. Error bars, s.d. (C) Relationship between irradiation light intensity and bioluminescence variation. The graph shows bioluminescence increasing ratios after light irradiation through the pinhole arrays. Light intensities were indicated under the graph. "100\%" shows the condition of intensity set in Figure 6, 7 and 8. Error bars, s.d. (D) Temporal variation of background bioluminescence intensities with repeated light irradiation. The irradiation condition was the same as that in Fig. S4B. Error bars, s.d. 

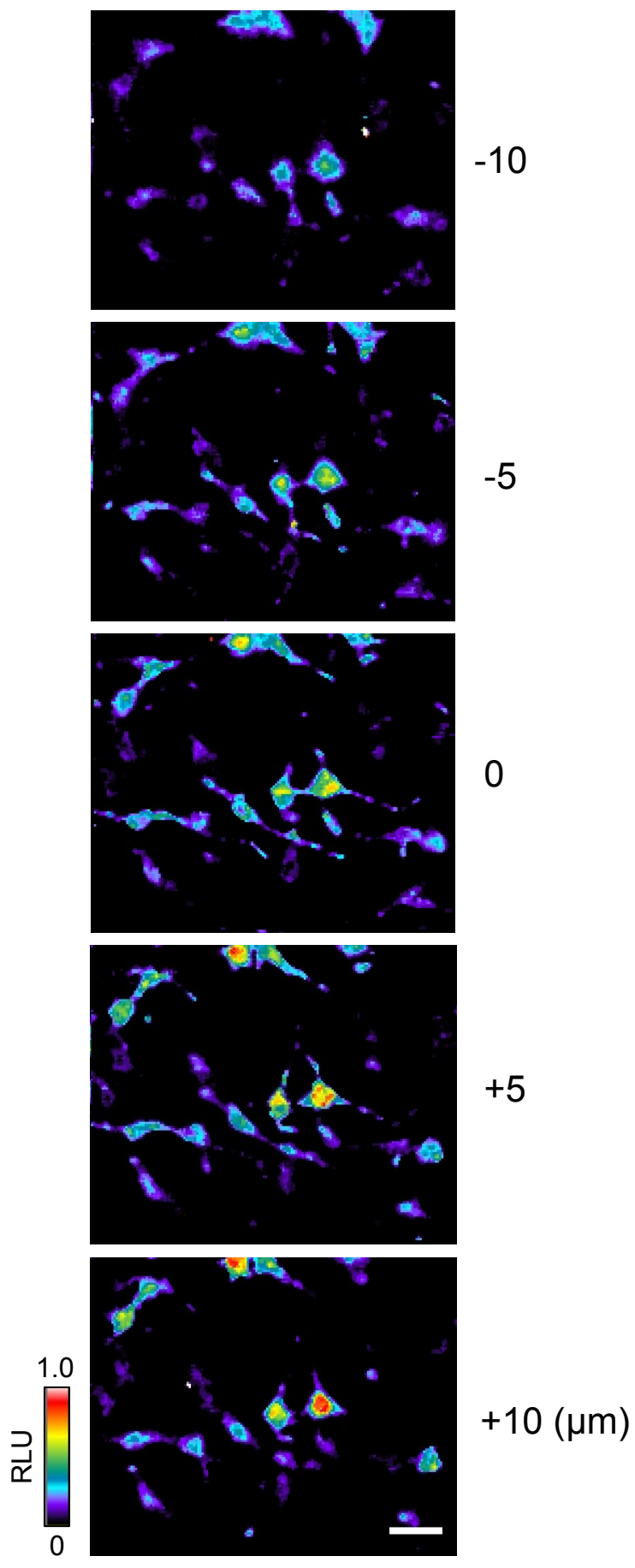

Figure S5. Pseudo-color images of bioluminescence from living cell layers coated with extracellular matrixes. The original data were from Fig. 7B. Scale bar, $50 \mu \mathrm{m}$. 
A

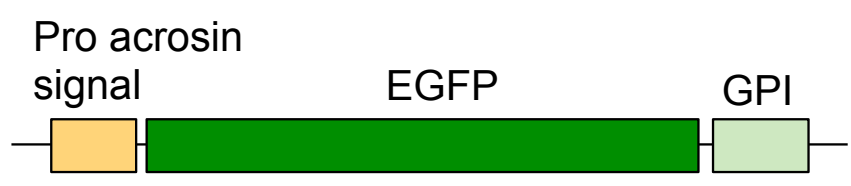

$\mathrm{B}$

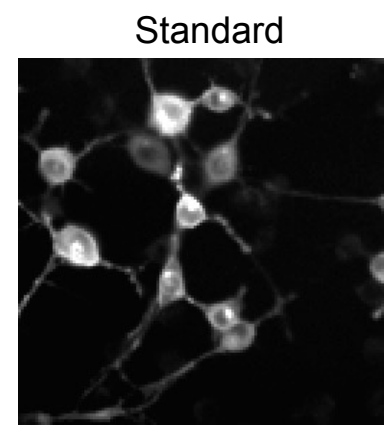

\section{Pinhole-based}

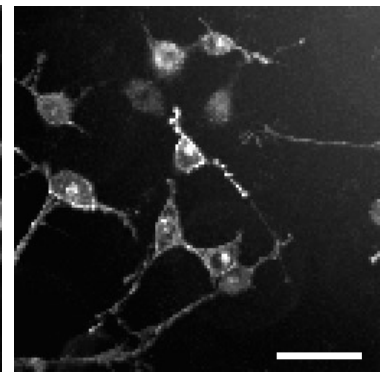

C

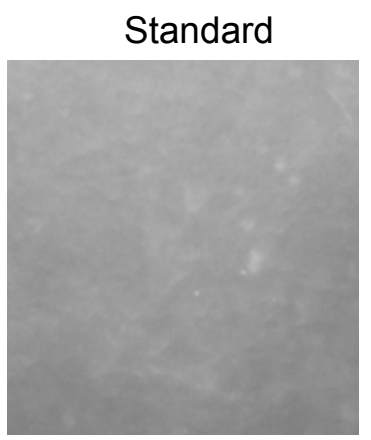

Pinhole-based

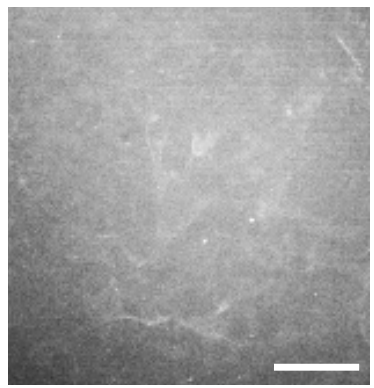

Figure S6. Scheme of the construct and fluorescence images of the extracellular matrix.

(A) Schematic of membrane-targeting EGFP. EGFP was connected with the proacrosin signal and GPI anchor. Amino acid sequences at connection sites were the same as those used in the NanoLuc probe. (B) Confocal fluorescence image of the EGFP in NIH3T3 cells. The left image (standard) shows a fluorescence image of the cells acquired using the confocal bioluminescence imaging system without pinhole arrays. The right image (pinhole-based) was acquired using the same system with pinhole arrays. Scale bar, $50 \mu \mathrm{m}$. (C) Fluorescence images of the EGFP in NIH3T3 cells coated with collagen and fibronectin. The right and left images were obtained using the bioluminescence imaging system with and without pinhole arrays. Scale bar, $50 \mu \mathrm{m}$. 


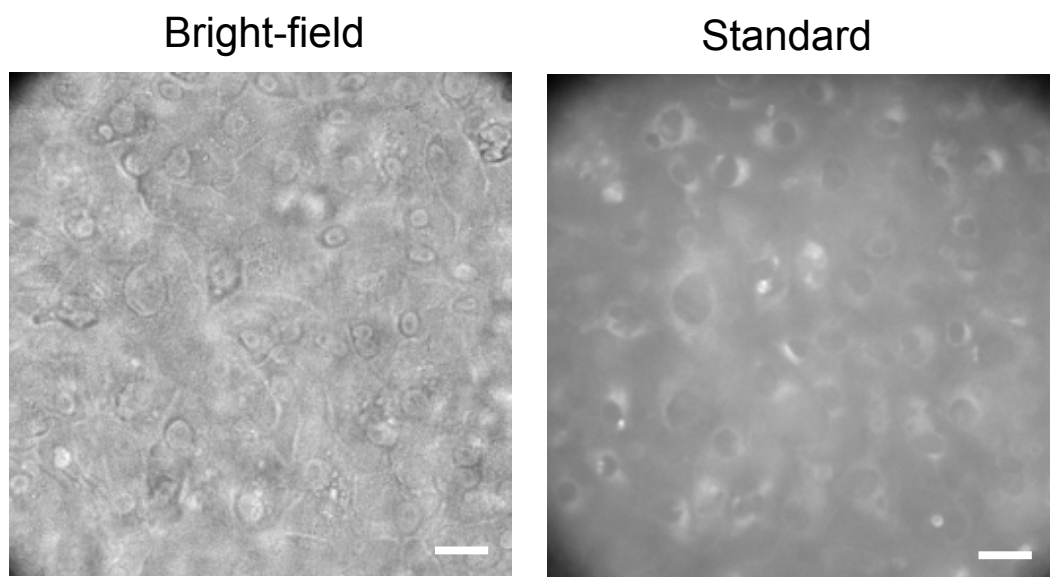

Figure S7. Fluorescence images of EGFP in an epidermal tissue. NHEK cells including EGFP were cultured on a cell-culture insert for 7 days. Left shows the bright field image. Right shows the confocal fluorescence image. Scale bar, $50 \mu \mathrm{m}$. 


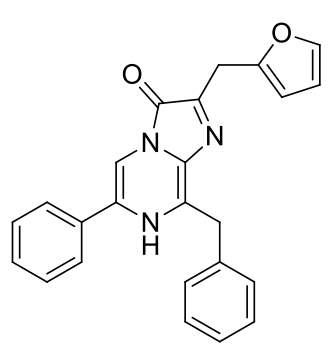

1

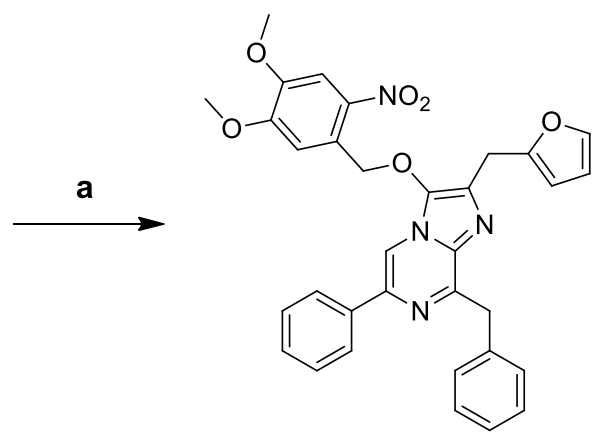

2

Scheme S1. Synthetic route of caged furimazine: (a) 4,5-dimethoxy-2-nitrobenzyl bromide, $\mathrm{K}_{2} \mathrm{CO}_{3}$, DMF, r.t. Furimazine (1) was synthesized according to a previous report. ${ }^{18}$ To a solution of 1 (3.0 mg, $7.8 \mu \mathrm{mol}, 1 \mathrm{eq})$ and potassium carbonate (10 mg, $72.4 \mu \mathrm{mol}, 9.2 \mathrm{eq})$ in anhydrous DMF (1.0 mL) was added dropwise 4,5-dimethoxy-2-nitrobenzyl bromide (11 mg, $39.8 \mu \mathrm{mol}, 5.1 \mathrm{eq})$ at $0{ }^{\circ} \mathrm{C}$. The mixture was stirred at r.t. under argon for 4 hours, then the solvent was evaporated. The residue was purified by preparative thin-layer chromatography ( silica gel, $\mathrm{n}$-hexane/EtOAc $=2 / 1)$ to give $2(2.3 \mathrm{mg}, 51 \%) .{ }^{1} \mathrm{H}$ NMR $\left(400 \mathrm{MHz}, \mathrm{CDCl}_{3}\right): \delta$ $8.05(\mathrm{~s}, 1 \mathrm{H}), 7.91-7.88(\mathrm{~m}, 2 \mathrm{H}), 7.75(\mathrm{~s}, 1 \mathrm{H}), 7.61-7.58(\mathrm{~m}, 2 \mathrm{H}), 7.46-7.42(\mathrm{~m}, 2 \mathrm{H})$, 7.39-7.35 (m, $1 \mathrm{H}), 7.32-7.26(\mathrm{~m}, 2 \mathrm{H}), 7.23(\mathrm{~s}, 1 \mathrm{H}), 7.22-7.18(\mathrm{~m}, 1 \mathrm{H}), 6.27(\mathrm{dd}, J=3.1 \mathrm{~Hz}$, $1.9 \mathrm{~Hz}, 1 \mathrm{H}), 6.12(\mathrm{dd}, J=3.1 \mathrm{~Hz}, 0.6 \mathrm{~Hz}, 1 \mathrm{H}), 5.46$ (s, $2 \mathrm{H}), 4.60(\mathrm{~s}, 2 \mathrm{H}), 4.21(\mathrm{~s}, 2 \mathrm{H})$, $3.97(\mathrm{~s}, 3 \mathrm{H}), 3.93(\mathrm{~s}, 3 \mathrm{H})$; HRMS (m/z): $[\mathrm{M}+\mathrm{Na}]^{+}$calcd. for $\mathrm{C}_{33} \mathrm{H}_{28} \mathrm{~N}_{4} \mathrm{NaO}_{6}, 599.19011$; found, 599.19003. 